\title{
IPCRG International Scientific Conference 5th June 2009, Stansted airport, UK Abstracts selected for the $P C R J$
}

\section{Pilot study of a population cohort for the study of COPD and OSAS in the Balearic population: The PULSAIB study Román $M$, Yáñez A, Renom F, de la Peña M, Gómez A, Duro R, Soriano JB}

Atención Primaria Ibsalut Fundación Caubet-Cimera

Aim: There are no population studies that describe the natural history of chronic obstructive pulmonary disease (COPD) and obstructive sleep apnea syndrome (OSAS). Their frequency and distribution in the Balearic Islands are unknown. We conducted a pilot study of a future population cohort in the Balearic Islands, the PULSAIB study, to investigate the natural history of COPD, OSAS, and other common chronic diseases.

Method: Cross-sectional study. 350 participants 30 years and older, in two areas, rural and urban. Sociodemographic and clinical questionnaires, forced spirometry with PBD test, portable polygraphy, EKG, and blood extraction were conducted.

Results: The participation rate was 53\%: 175 participants from the municipality of Bunyola and 130 from the area of Son Pizà Health Center in Palma. Prevalence of COPD ( $\mathrm{FEV}_{1} / \mathrm{FVC}$ ratio $\left.<0.70 \mathrm{PBD}\right)$ was $12.8 \%(95 \% \mathrm{Cl}$ $8.5 \%-18.2 \%$ ), $20 \%$ in men and $5 \%$ in women. Prevalence of OSAS (apneahypopnea index (AHI)> 10/hour) was 35.9\% (95\% Cl 25.3\%-47.6\%), $42 \%$ in men and $29 \%$ in women.

Conclusion: We conclude that setting up a cohort study in the Balearic population is doable, expanding in number and following up in time the participants of this pilot study, all aimed to investigate the natural history of the two most common chronic respiratory diseases, COPD and the OSAS. Conflict of interest and funding: Fundings by Balearic Goverment 12-2006 Research Emerging Groups.

2. 'A way of life'. Findings from a longitudinal qualitative study of people with end-stage COPD.

Pinnock H, Murray S, Worth A, Porter M, MacNee W, Kendall M, Levack P, Sheikh A.

Allergy and Respiratory Research Group, Centre for Population Health Sciences: GP Section, University of Edinburgh

Aim: Globally, there is increasing interest in the provision of palliative care services for people with COPD (IPCRG Opinion sheet 4). In the UK, primary care clinicians are encouraged to include people with very severe COPD on palliative care registers to facilitate provision of end-of-life care.

Method: We conducted serial in-depth interviews over 18-months with people with severe COPD and their nominated informal and professional carers to identify their clinical, information, psycho-social and spiritual needs. Interviews were transcribed and analysed thematically.

Results: 21 patients (7 died during the study) and their social and professional carers, provided 130 interviews. Severe symptoms causing major disruption to daily life were described in the context of deprived social circumstances which patients often felt powerless to change. Particularly striking was that COPD was perceived as a 'way of life' rather than an 'illness'. The insidious development of symptoms over a lifetime of smoking made it impossible for patients to describe the beginning of their condition, although significant milestones (e.g. diagnosis, first admission) were highlighted. Clinicians had reservations about identifying the 'moment' when palliative care became appropriate in a condition which typically spanned decades.

Conclusion: Acceptance of COPD as 'a way of life' by patients, in the context of seemingly insurmountable social difficulties, has important implications for healthcare professionals. Identifying a time point for inclusion on a palliative care register may be impossible and potentially counter-productive if it obstructs provision of holistic care.

Conflict of interest and funding: Chief Scientist's Office, Scottish Government. HP is supported by a PCRCA from the Chief Scientist's Office of the Scottish Government.

Published or submitted elsewhere: Conference submission: European Respiratory Society 2009.

3. Technique training does not improve the ability of most patients to use pressurised Metered Dose Inhalers (pMDI)

Hardwell A, Barber V, Hargadon T, Levy M

National Services for Health Improvement, Dartford, Kent

Aim: pMDI technique should be tested in all asthmatic patients. ' We assessed uncontrolled asthma patients ability to learn to use a pMDI using the Aerosol Inhalation Monitor (AIM, OVitalograph) which tests inspiratory flow, synchronisation and breathholding.

Method: Patients were clinically reviewed by trained asthma nurses, according to practice protocols. After agreeing a proxy measure of asthma control (prescribed rescue bronchodilators), selected patients completed postal symptom questionnaires. (RCP $3 \mathrm{Q})^{1}$ Symptomatic patients were invited for a clinical review, including inhaler technique. Those patients using pMDIs had at least two assessments. Technique education was provided after testing if appropriate.

Results: 2123/8843 (24\%) symptomatic patients from 100 practices caring for 30779 asthmatics (prevalence 6.9\%) were assessed by nurses between 1/4/2008 and 30/6/2008. (Data available for 2112 patients). 1291/2112 (61\%) were using pMDI's (mean age 52 years; SD 21.37). 685 (55.4\%) and $368 / 1236$ (29.8\%) were in BTS steps two and three respectively. 1092/1275 (85.6\%) using pMDIs, failed the first AIM test. After instruction the number of patients able to use their pMDIs correctly increased after the second (129 to 260 of 1197 patients, $p<0.01$ ) and third (61 to 181 of 528 patients, $p<0.01$ ) tests. 909/1197 (76\%) and 323/527 (61.3\%) patients tested twice and three times respectively, failed on these subsequent attempts, despite instruction. No effect of age and BTS step on these outcomes.

Conclusion: Despite training a significant majority of symptomatic asthma patients are unable to use pMDIs correctly.

Reference:

1. Douglas G. et al. Thorax 2008; 63(Suppl. 4):iv1-iv121.

Conflict of interest and funding: The EACS service is an independent nurse service sponsored by TEVA UK Limited.

Published or submitted elsewhere: Conference submission: BTS Winter Conference 2008 


\section{Effect on health care costs of an asthma education intervention in primary care \\ Cave A, Bhanji N, Makarowski C \\ Department of Family Medicine, University of Alberta, Canada}

Aim: To assess the impact on direct health care costs of asthma education delivered in the family physician's office.

Method: A site-randomised, unblinded controlled study of an educational intervention for asthma. 85 Family Physicians (FPs) in Alberta invited asthma patients to participate. 200 of 250 were enrolled. Half the practices were allocated to control group for six months and half to intervention. Both groups were assessed at 0,6 and 12 months. Control group completed baseline spirometry, and study questionnaires, and received an educational brochure. The intervention group also received three 40 minute education sessions from a certified asthma educator. The self reported Edmonton Asthma Behaviour Scale (EABS), included a question on unplanned health care use over the last three months.

Results: 58 subjects in the control group and 60 in the intervention group have completed six month assessments. Control group costs (by EABS scoring) fell from $\$ 115.6$ to $\$ 61.9$ per patient $(47 \%)$ and the intervention group costs fell from $\$ 178.2$ to $\$ 107.9$ per patient (39\%). Medication costs are not included

Conclusion: Asthma assessment and patient evaluation by a certified asthma educator in the FP's office can reduce direct health care costs for these patients. Conflict of interest and funding: Financial Support: Alberta Heritage Foundation for Medical Research, Alberta Strategy for The Management of Asthma (ASTHMA), Astra-Zeneca, GSK Canada, Nycomed and Boehringer Ingelheim. AC has presented and advised for each of these companies. NB and CM have no conflicts of interest. We prefer an oral presentation.

\section{Impact of asthma education on patient quality of life Cave A, Makarowski C, Ahmadi E}

Department of Family Medicine, University of Alberta, Edmonton, Canada

Aim: Providing asthma education in the Family Physician's (FP) office expands the model of primary care in Canada and provides an expanded role for health professionals in the primary care team. Our objective was to evaluate the impact of asthma education in FP's offices on patient quality of life.

Method: In a site randomized, unblinded controlled study of education for asthma, 85 FPs from 28 practices identified asthma patients and invited them to participate. 250 asthma patients were screened and 200 were enrolled.(35 children). Half the practices were allocated to a control group and half to the intervention. Both groups were assessed at 0,6 and 12 months. Subjects in the control practices completed spirometry and baseline asthma assessments and received an educational brochure. The study group received an additional three sessions of one-to-one education (totaling two hours) from the educators. One of the outcomes assessed was quality of life using Juniper's Asthma Related Quality of Life Questionnaire.

Results: Data for 128 adult subjects who completed the six months assessments shows statistically significant improvements in quality of life for both groups. The intervention group, with extra teaching, shows a greater improvement but this difference is not statistically significant.

Conclusion: Asthma assessment by certified educators, can improve quality of life for patients with asthma when provided in FP offices. Personalised, patient-centred education can add to this effect.

Conflict of interest and funding: Financial Support: Alberta Heritage Foundation for Medical Research, Alberta Strategy for The Management of Asthma (ASTHMA), Astra-Zeneca, GSK Canada, Nycomed and BoehringerIngelheim.

\section{Different factors affecting asthma control in Ireland \{Helping Asthma in Real People (HARP) Study\}}

Bajwa Joseph M, Price D, Sims E, Haughney J, Keeley J, Shanahan E, Holohan J, Ryan D

Research in Real Life

Aim: HARP is an asthma audit and review service developed by the International Primary Care Research Group (IPCRG) in conjunction with Asthma Society of Ireland and Optimum Patient Care (OPC) using electronic patient data and/or questionnaire to inform and facilitate the flow of information between the patient and physician. The aim was to analyse questionnaire results from patients' correspondence to determine reasons for poor asthma control.

Method: Currently in Ireland, there is no standard treatment/ disease coding system used. Therefore, patients were identified by asthma treatment prescription codes as follows:

Patients identified by treatment data alone

- No data extracted due to the inconsistent coding of clinical data and subsequent difficulty in analysis

- Questionnaires sent to all identified patients

- Reports generated on all questionnaire respondents

GP review involved asking patients to complete asthma control test (ACT) including the Royal College of Physician (RCP) asthma score questionnaires. Results: Fifteen practices were audited, a total of 3973 number of patients were mailed of which 1444 patients responded, and a response rate of $36.3 \%$ was achieved. 894 respondents classified as uncontrolled 550 respondents classified as controlled. Summary of results:

1. Of the 1444 respondents, $61.9 \%$ were classified as uncontrolled.

2. Respondents with uncontrolled asthma were more likely to have significant rhinitis.

3. $25.8 \%$ of respondents with uncontrolled asthma had significant rhinitis compared to only $10.6 \%$ of respondents with controlled asthma.

4. $48,7 \%$ of respondents with uncontrolled asthma compared to $35.1 \%$ of those with controlled asthma reported low adherence to therapy.

Conclusion: The large majority of uncontrolled respondents had comorbid factors hindering asthma control which the HARP project is now addressing. Conflict of interest and funding: None.

\section{Variation in COPD management in 100 UK practices Price D, Sims E, Jones R, Freeman D, Ryan F \\ Research in Real Life}

Aim: Despite the publication of several sets of COPD guidelines there is still a high level of variation in the management and treatment of COPD in primary care. This study was designed to quantify the difference between treatment of COPD patients in primary care and recommended treatment as provided by NICE and BTS management guidelines.

Method: Data of all diagnosed COPD patients were collected from 100 UK practices. In order to prevent bias of the results in practices where routine medical data was not in a form where it could be readily collected all COPD patients were sent a questionnaire including validated instruments asking about their perception of their condition. Questionnaire data (where available) was then matched with the corresponding medical record allowing each patient's situation and therapy to be compared to treatment recommended by the guidelines.

Results: Routine data from 10538 COPD patients was collected and 4386 questionnaires were returned giving a response rate of $41.6 \%$.

$22.1 \%$ of patients were found to have at least one drug treatment out of line with recommendations.

- $\quad 37.8 \%$ of patients had at least one management or therapy (other than drug treatments) out of line with the guidelines.

- $\quad 35.3 \%$ of patients were found to require either further assessment, change in therapy or other complimentary therapy to bring management of their condition in line with recommendations.

Very high levels of inter-practice variation were found. For example $27 \%$ 
of practices had $\geq 80 \%$ of patients MRC scores recorded whilst $18 \%$ of practices had $\leq 30 \%$ of patients MRC scores recorded.

Conclusion: There are high levels of inter-practice variation as well as high levels of deviance from guideline COPD treatment and management overall. Conflict of interest and funding: BI.

\section{Canadian family physician preparedness for management of TB}

Cave AJ, Salehar M, Parameswaran S, Kaplan A.

Department of Family Medicine, University of Alberta, Edmonton, Canada

Aim: The preparedness of primary care physicians for early detection and management of TB is crucial in preventing spread in the community. Studies in USA and Pakistan have found a low level of familiarity with current TB management in primary care.

Method: A survey was mailed during March 2008 to 950 affiliated members of the Family Physicians Airways Group of Canada. Email warnings of the survey were sent one week before mail out and a reminder one week after mail out. A single sheet survey with questions concerning physician demographics, experience with $T B$, confidence in managing $T B$ and knowledge testing questions was mailed.

Results: There was a $27 \%$ response rate. Membership is heavily weighted in favour of Ontario physicians and so data from this province was analysed separately ( $\mathrm{N}=172)$. Of these, $8.7 \%(15)$ were trained in areas of the world where TB is endemic, 78\% (132) practiced in an urban area and 69\% (117) had less than ten patients with TB.

Graduating outside North America ( $p=0.0009)$, coming from an endemic area $(p=0.019)$ or seeing more than 10 TB patients $(p<0.0001)$ resulted in higher confidence with managing TB but none of these factors showed significant differences in management knowledge scores.

Conclusion: Graduating in North America and having little exposure to patients with TB lowers preparedness to manage TB in primary care. Education should be targeted to those physicians.

Conflict of interest and funding: Funding was provided by the Public Health Agency of Canada. No author has a conflict of interest.

Published or submitted elsewhere: Conference submission: Nonreferenced poster presentation at International Union Against TB and Lung Disease (IUTBLD) regional meeting, Vancouver, Feb 2009.

\section{Chronic Obstructive Pulmonary Disease Patient Action Plan for Acute Exacerbations: evaluation study Kaplan A, Hernandez P, Bourbeau J, Power B, Gupta S, Goubanova E \\ Family Physician Airways Group of Canada}

Aim: Acute exacerbations of COPD (AECOPD) are the most frequent cause of medical visits, hospital admissions and death among patients with COPD. The recent CTS guidelines recommended appropriate prevention strategies and early identification and treatment of exacerbations. The purpose of this evaluation study was to assist the Canadian Thoracic Society in assessing the effectiveness of its guidelines implementation tools.

Methods: The CTS COPD Action Plan was developed and distributed to the sample of GPs, respiratory educators and pharmacists with an accompanying introductory letter and a questionnaire.

Results: Based on the feedback received to date, respondents indicated strong positive perception of the tool effectiveness in managing exacerbations. If implemented appropriately, respondents expect positive outcomes such as: a decrease in emergency department visits; and a shorter symptom recovery time which will improve health-related quality of life. Majority of respondents (96\%) expect positive outcomes from implementing the COPD Action Plan. They place their most positive evaluation of Action Plan outcomes on decreasing the ER visits and hospital admissions. Majority of respondents expect their patients to promptly use prednisone (96\%) and antibiotic ( $81 \%$ ) if supported by patient education and a proper follow-up. Conclusion: All three groups had identified strong need for future professional development activities and resources. Overall, based on different priorities and needs of respondents from three groups surveyed at the evaluation phase (GPs, RTs and pharmacists), advances in CME/CHE may help in the development of multidisciplinary workshops serving the common needs of health care professionals across the professional and geographic boundaries.

Conflict of interest and funding: The study was funded by the Canadian Thoracic Society. Authors declared no conflict of interest.

Published or submitted elsewhere: Conference submission: Canadian Society Respiratory Therapy Conference, May 2009.

\section{Incidence of asthma and accuracy of diagnosis in the Portuguese Sentinel Practice Network Correia-de-Sousa J, Silva ML, Almada-Lobo F, Yaphe J School of Health Sciences, University of Minho, Braga, Portugal}

Aim: In 1999 the Portuguese Sentinel Practice Network decided to study the incidence of new cases of asthma presented to members of the network over a four year period. This report presents data from this network study and explores the question of diagnostic accuracy for the diagnosis of asthma by network members.

Method: Data were collected by 54 network physicians in sentinel practices in over a four year period in 2000-2003. All patients consulting with their Family physician with complaints suggestive of asthma were enrolled in the study. Data were collected on a standardized data reporting form. All forms were collected on completion and data were coded and entered for analysis. Frequencies of symptoms, diagnoses of asthma and place of diagnosis were tabulated. Diagnostic accuracy was computed by dividing the rate of asthma diagnosis by family physicians by the true rate as determined by established diagnostic criteria.

Results: 128412 patients were seen in this study. Asthma was diagnosed in 310. The true incidence was 2.02/ 1000/ year as determined by accepted criteria giving an accuracy rate of $84 \%$.

Conclusion: The incidence of asthma was lower than expected in this population if only the clinical diagnoses of family physicians are used. The incidence approaches rates in the published literature if accepted criteria are used. Outcomes for asthma patients may be improved in this population if accepted criteria are used widely. Educational efforts should be focused in this direction.

Conflict of interest and funding: No conflict of interests; funding through Portuguese Government bodies.

Published or submitted elsewhere: Publication reference: Accuracy of diagnosis of new cases of asthma by the Portugal Sentinel Network; Conference submission: WONCA Conference, Amsterdam 2004, only partial data presented.

\section{Long-term results of integrated COPD management in primary care: the Kroonluchter Program Chavannes N, Gussekloo J \\ Leiden University Medical Center, Netherlands}

Aim: Integrated Disease Management (IDM) of primary care COPD-patients has been demonstrated to improve exercise tolerance and quality of life after one year, but longer-term results are currently lacking.

Method: Since 2006, the Kroonluchter IDM-program has treated 216 primary care patients with mild to moderate COPD. Prospective six-minute walking distance (6MWD)results of patients reaching 24 months of follow-up were analyzed using paired-samples T-tests.

Results: Patients that completed follow-up had a mean FEV 1 of $65 \%$ predicted and were aged 68 years on average. The mean improvement compared with baseline on the 6MWD was 78m [95\% Cl 49-106] $p<0.001$. Up to $62 \%$ of patients reached and maintained the MCID of $54 \mathrm{~m}$ on the 
Abstracts

6MWD during 24 months of follow-up.

Conclusion: Integrated Disease Management can lead to long-term improvements in primary care COPD patients, the majority of whom reach and maintain clinically relevant differences in exercise tolerance.

Conflict of interest and funding: None.

Published or submitted elsewhere: Conference submission: ERS Congress 2009.

12. Knowledge of asthma guidelines: Results of a web-based 'Test your Knowledge' quiz

Pinnock H, Small I, McArthur R, Holmes S, Levy ML for the GPIAG Education Committee

Allergy and Respiratory Research Group, Centre for Population Health Sciences: GP Section, University of Edinburgh

Aim: Each update of the British Thoracic Society and Scottish Collegiate Guideline Network (BTS-SIGN) guideline for the management of asthma has been widely disseminated. The General Practice Airways Group (GPIAG) survey aimed to test the knowledge of visitors to the web-based 2008 guideline summary.

Method: Web-site visitors were invited to complete an anonymous questionnaire, comprising eleven multiple choice questions. Responses were marked as correct or incorrect giving a score out of 11. In addition to descriptive statistics, responses between groups were compared using Chisquared or independent sample t-tests.

Results: The 413 respondents (96 (23.2\%) general practitioners (GPs), 237 (57.4\%) primary care nurses (PCNs) $38(9.2 \%)$ secondary care nurses (SCNs) and $42(10.2 \%)$ other healthcare professionals) answered less than half the questions correctly. The mean score was 5.2 (SD 2.56): 24 respondents answered all questions correctly; three scored no correct answers. GP scores were significantly lower than primary care nurses $(\mathrm{GP}=4.6$ vs $P C N=5.5$. mean difference $-0.97(95 \% \mathrm{Cl}-1.6$ to -0.36$) \mathrm{p}=0.002)$ There was no difference between the scores of PCN and SCN, or between GPIAG and non-GPIAG members. There were differences between professional groups in accuracy of response to individual questions which to some extent reflected their roles within UK practices.

Conclusion: There is still an urgent need to promote the key messages from the BTS-SIGN asthma guidelines. Although compliance with guidelines is multifactorial, improving healthcare professionals' knowledge of asthma is an important pre-requisite.

Conflict of interest and funding: The GPIAG project was funded by educational grant from GlaxoSmithKline. HP is supported by a PCRCA from the Chief Scientist's Office of the Scottish Government.

\section{The challenges of recruiting for a primary care trial Malhotra S, Musgrave S, Price D, Pinnock H, Ryan D University of Aberdeen Scotland}

Aim: We aimed to recruit 312 participants to our pragmatic trial of using mobile technology to improve poorly controlled asthma. We here report the challenges of recruitment.

Method: We interrogated the computerised databases of participating practices using dedicated software, identifying patients 12 years and over with poorly controlled asthma (Asthma Control Questionnaire (ACQ) >1.5) After GP check for suitability, all identified patients were sent a postal invitation. Those expressing interest were pre-screened by phone to check their asthma control and that they had a suitable telecommunication package. Potentially eligible patients were booked for trial recruitment visit where inclusion/exclusion criteria were confirmed.

Results: 32 practices participated (combined list size 311,926 patients). Computer interrogation identified 13,101 patients, of whom 1,020 were excluded by the GPs as inappropriate, 12,081 invitations posted, 1,016 $(8.4 \%)$, patients expressed an interest and were pre-screened by telephone. 470 were excluded because of good asthma control, 69 because of telecommunication package incompatibility and 84 for other reasons. Of 391 (3.2\%) potentially eligible patients booked for trial recruitment visit $47 \mathrm{did}$ not attend, 5 had COPD, 3 had moved, 37 were no longer poorly controlled leaving a total of 288 patients for randomisation, $2.4 \%$ of those invited.

Conclusion: Understanding the barriers to recruitment could facilitate a more efficient process. Better coding of routine data in primary care and more sophisticated software tools could improve the focus of computer searches. Patient friendly information packs and invitations should encourage response; Pre-screening effectively excluded ineligible participants but may act as an intervention. Incompatibility between telecommunication packages further challenged recruitment.

Conflict of interest and funding: Conflicts of interest: None; Funding: Asthma UK. 